\title{
Time Dependent Diffusion as a Mean Field Counterpart of Lévy Type Random Walk
}

\author{
D.A. Ahmed *, S. Petrovskii \\ Department of Mathematics, University of Leicester, University road, Leicester, LE1 7RH, UK
}

\begin{abstract}
Insect trapping is commonly used in various pest insect monitoring programs as well as in many ecological field studies. An individual is said to be trapped if it falls within a well defined capturing zone, which it cannot escape. The accumulation of trapped individuals over time forms trap counts or alternatively, the flux of the population density into the trap. In this paper, we study the movement of insects whose dynamics are governed by time dependent diffusion and Lévy walks. We demonstrate that the diffusion model provides an alternative framework for the Cauchy type random walk (Lévy walk with Cauchy distributed steps). Furthermore, by calculating the trap counts using these two conceptually different movement models, we propose that trap counts for pests whose dynamics may be Lévy by nature can effectively be predicted by diffusive flux curves with time-dependent diffusivity.
\end{abstract}

Keywords and phrases: anomalous diffusion, lévy walk, trap counts, insect monitoring, foraging

Mathematics Subject Classification: 82B41, 60K35, 35Q92

\section{Introduction}

In ecological studies, the dynamics of a population is usually best described in terms of the population density or population size. Ecological monitoring programs require estimates of pest species abundance. Important information about population numbers (or trap counts) can be translated and used to make decisions with regards to pest control, costs and pesticide application [67]. Chemical pesticides have their obvious drawbacks in additional operator costs, wheeling damage to the crop and general agricultural produce damage. In another ecological context, accurate information about population abundance is required to trace the spread of harmful invading species. In the case of insects, their density is usually estimated based on trap counts $[45,46]$. One way to look at the dynamics of trap counts is to simulate them by modelling the movement of each individual in the field. This approach is known in the literature as an individual-based model [71]. Furthermore, experimental trap counts can be simulated via computer algorithms where the properties of the individual movement are fully determined by the step distributions and turning angle distribution. A comprehensive mathematical framework for individual based modelling can be found in [46] and also presented below in $\S(2.2)$.

\footnotetext{
${ }^{*}$ Corresponding author. E-mail: daa119@outlook.com
} 
In foraging theory it is often assumed that the step lengths of a forager have a characteristic scale. The assumption has led to investigations of Gaussian, Rayleigh and other classical distributions with well defined variances. It is observed experimentally that step lengths tend to follow long tailed power law type distributions, formally known as Lévy walks. Movement models which incorporate Gaussian distributed steps fail to explain these experimental observations $[10,11,24,55,66,70]$. Lévy walks are characterized by a probability density function (p.d.f),

$$
\phi\left(l_{i}\right) \sim l_{i}^{-\mu}
$$

with relative importance given to the exponent $1<\mu<3$, where $l_{i}$ is the step length of the $i^{\text {th }}$ step. This characterization introduces a natural parameter $\mu$, such that we have a family of distributions [72]. Due to the central limit theorem, the Gaussian is the stable distribution for the special case $\mu \geq 3$.

The best statistical strategy to adopt in order to search efficiently for randomly located objects known as target sites is provided by a movement model with Lévy distributed step lengths. In particular when the target sites are sparse an inverse square power-law distribution $\mu=2$ of step lengths provides an optimal searching strategy. The optimality is dependent upon searchers being able to profitably revisit targets. The strategy has been tested and applied to experimental foraging data of various species (insects, mammals and birds) with relevant consistency. The theoretical arguments and numerical simulations provided by [72] show that $\mu=2$ is the optimal value for a search in any dimension. With this biological relevance, our study comprises of Lévy walks with exponent $\mu=2$, in particular Cauchy distributed steps.

General theoretical models of animal movement are stochastic in nature. In some cases, these models are well known to be effectively approximated via diffusion models. For instance, random walks (1.1) with $\mu \geq 3$ are often approximated by advection diffusion type models $[9,43,71]$. The diffusion counterpart facilitates parametrization and analysis of movement models. Diffusion approximations are simpler to interpret in comparison to stochastic models of movement (random walks) due to the aggregation of parameters of a simulation model (e.g distributions of turning angles, step lengths) into relevant summary statistics (e.g diffusion coefficient). This provides effective comparisons to be made amongst studies [71]. In some simple cases, analytical solutions can be written or even sometimes exact solutions. A concise introduction to the mathematical theory behind the random walk and its relation to diffusive processes is given by Codling et al. [12]. Our aim in this paper is to demonstrate that the diffusion approximation in fact is not limited to the case of Brownian motion $(\mu \geq 3)$ and time dependent diffusion is an accurate approximation to the Lévy type random walk with Cauchy step distribution. This is done in the context of trapping, where simulated trap counts are modelled by diffusive flux.

\section{The mean field and individual based description}

Anomalous diffusion has been known since the treatment of accelerated type turbulent flows [56]. The hallmark of an anomalous diffusive process is such that the mean squared displacement is proportional to $t^{2 H}$ with $H \neq \frac{1}{2}$, [58,73,74]. Theoretical investigations instigated by Scher and Montroll [58] in their description of dispersive transport in amorphous semi-conductors, found that traditional methods based on Brownian models failed. In the physical sciences, over the last half century, anomalous diffusion models have been developed with important contributions from Weiss and Rubin [76] and Shlesinger [62]. Currently, the list of complex systems displaying anomalous diffusion behaviour is quite extensive $[4,35,59,75,77]$, and the problem of trapping (more commonly known as system flux) is well known in physics. However, most studies are concerned with large-time asymptotics whilst for insect monitoring short time dynamics are of primary concern $[46,50]$.

In the context of ecological pest monitoring, a consistent theory and robust computational algorithms exist in the case where insects perform Brownian motion [50]. There is increasing evidence that more generally, animals may exhibit a more complicated type of movement pattern which deviates from the usual Brownian movement (standard diffusion) such as Lévy walks [29,33,61], however a theory linking 
trap counts to population density is severely lacking. The common approach used in early studies for population dispersal and basic movement models were entirely based on random walks and diffusion $[21,40,49,64]$, effectively providing good agreement with empirical data [30,41,71]. Although a diffusion approach has been used successfully in spatial ecology $[9,27,34,42,47,60]$, it has also been recognized to sometimes provide an oversimplified description of individual movement. Correspondingly, recent theoretical studies tend to denounce the random walk framework as inappropriate.

The Mean Field Approach (MFA) and Individual Based Model (IBM) have been shown to be consistent in the context of estimating trap counts $[46,50]$, for a system whose movement dynamics are of a Brownian nature, corresponding to the constant diffusion case. Individual based models provide information on the fluctuations in the system and provide a basis for the study of the stochasticity of the system. The approach allows trap counts to be simulated directly for any given initial distribution, and for any generic movement pattern whose step lengths are prescribed by a probability distribution. Henceforth, real-world problems on pest control can be imitated in a simulation setting. Although effective, this treatment is limiting in the sense that conclusions cannot be generalized for varying parameters. Alternatively, deterministic systems and mean field approaches reveal parameter dependencies and the generalized behaviour of the dynamics $[1,12,47,71]$. It is also known that analytical solutions exist to successfully describe time dependent diffusive behaviour (see Appendix A) e.g in the 1D case with semi-infinite domain [14]. Therefore, it must be appreciated that both approaches are very important for the study of pest movement and trap counts, to facilitate broader interpretation.

\subsection{Mean field approach (MFA)}

It is well known that the simple diffusion equation has extensively been used to model the spatio-temporal population density $u(x, t)$ of moving pests. The model assumes that the environment is constant, homogeneous and that all individuals are identical and move randomly [30,42]. The 1D governing diffusion equation reads,

$$
\frac{\partial u}{\partial t}=D \frac{\partial^{2} u}{\partial x^{2}}, \quad 0<x<\eta, \quad t>0,
$$

defined over a spatial scale $0<x<\eta$ with time $t>0$ and diffusion coefficient $D$. For time dependent diffusive processes, $D=D(t)$ is explicitly dependent on time and non-constant. We prescribe the zero density condition $u(x=0, t)=0$ and no-flux condition $\frac{\partial u(x=\eta, t)}{\partial x}=0$ at the left/right hand boundaries, respectively. The biological significance of the reflective boundary at $x=\eta$ is such that there is no migration, and individuals can only exit the system through the boundary at $x=0$, consequently the population density can only decrease with time. The initial profile can be described by $u(x, t=0)=$ $N \delta\left(x-x_{0}\right)$ for a total population of $N$ individuals, released at a concentrated point $x=x_{0}$, more formally known as a Point Source Release (PSR). Here, $\delta$ is the Dirac delta function. Alternatively, for a homogenous system where individuals are uniformly distributed we have that $u(x, t=0)=U_{0}=\frac{N}{\eta}$. Numerical solutions can be obtained for (2.1) using a variety of techniques (see $[65,68]$ ). In particular, the implicit finite difference scheme is advantageous in comparison to explicit schemes since limitations such as the Courant stability criterion are relaxed $[1,44,68]$. However note that, the initial condition in the form of a PSR cannot be discretized, therefore these schemes fail to approximate such a condition. To overcome this, we introduce a Gaussian type formulation with small width $\sigma$, normalised over the finite domain [1],

$$
u(x, t=0)=\frac{N}{\zeta \sqrt{2 \pi \sigma^{2}}} \exp \left(-\frac{\left(x-x_{0}\right)^{2}}{2 \sigma^{2}}\right)
$$

which can be discretized. Here $\zeta=\int_{0}^{\eta} \frac{1}{\sqrt{2 \pi \sigma^{2}}} \exp \left(-\frac{\left(x-x_{0}\right)^{2}}{2 \sigma^{2}}\right) d x$ is a re-scaling constant used for conservation purposes to ensure that the total population $N$ lies within the domain $0<x<\eta$ at $t=0$, 
i.e $\int_{0}^{\eta} u(x, t=0) d x=\int_{0}^{\eta} \frac{N}{\zeta \sqrt{2 \pi \sigma^{2}}} \exp \left(-\frac{\left(x-x_{0}\right)^{2}}{2 \sigma^{2}}\right) d x=N=U_{0} \eta$. For sufficiently small width $\sigma$, successfully mimics the PSR ${ }^{1}$

The trap counts or the total flux of the system is defined as,

$$
J(t)=\left.\int_{0}^{t} D\left(t^{\prime}\right) \frac{\partial u\left(x, t^{\prime}\right)}{\partial x}\right|_{x=0} d t^{\prime},
$$

where $J(t)$ represents the number of individuals which exit the system after time $t$ i.e the total number caught or trapped. Since there is a reflective boundary at $x=\eta$, it is evident that the trap counts can only increase monotonously with time. Here, (2.3) assumes the 1D analogue of the Fickian hypothesis in isotropic media, that the rate of transfer of diffusing substance through unit area of a section is proportional to the concentration gradient measured normal to the section $[14,20]$. If the analytic form for $u(x, t)$ is unknown, $(2.3)$ cannot be evaluated and we resort to numerical integration techniques $[15,19]$.

\subsection{Individual based model (IBM)}

The dynamics of trap counts can also be studied using individual based models [25,71]. The basic idea is to simulate the movement in the field. Consider a browsing individual over the domain $0<x<\eta$, we denote $x=X_{i}, i=0,1,2, \ldots, s-1, s$ as the position after the $i^{t h}$ step where $s$ is the total number of steps executed. A standard observation technique usually makes it possible to record the pest position only at certain moments rather than continuously. The continuous time $t$ thus transforms into a finite set $\left\{t_{0}, t_{1}, t_{2}, \ldots, t_{s}\right\}$ and correspondingly, a path is mapped into a broken line $\left\{X_{0}, X_{1}, X_{2}, \ldots, X_{s}\right\},[71]$. Generally speaking, the time step $\Delta t_{i}=t_{i+1}-t_{i}$ can be different for different $i$. In order to avoid unnecessary complexity, we assume that $\Delta t_{i}=\Delta t=$ const and does not depend on $i$, so that $t_{i}=i \Delta t$. The absence of correlation between any two subsequent steps depends on the time step $\Delta t$ of the observations. The assumption that subsequent steps are uncorrelated, is not viable for a sufficiently small value of $\Delta t$. The movement direction of the preceding step is preferred for the next step, resulting in the expected values of direction to be centered about zero, [46]. The corresponding random process is known as the correlated random walk (CRW), [31]. Remarkably, however, the CRW attains the properties identical to Brownian motion when $\Delta t$ becomes sufficiently large [31]. We assume that the walk is isotropic (or unbiased, no-persistence) so that each individual is equally likely to move in each possible direction, which is totally independent of the direction at all preceding times. The process is effectively Markovian with respect to location $[12,75]$.

Over the interval $\Delta t$, the individual moves along a straight line from $X_{i-1}$ to $X_{i}$, thus executing a step $\Delta X_{i}=X_{i}-X_{i-1}$ with velocity $\mathbf{v}_{i}=\frac{X_{i}-X_{i-1}}{\Delta t}$, here $\Delta X_{i}<0$ corresponds to a step in the negative $x$ direction. The step length is defined as $l_{i}=\left|\Delta X_{i}\right|=\left|\Delta X_{i}-\Delta X_{i-1}\right|$, which is strictly non-negative. We assume that there is no correlation between any two subsequent steps $\left\langle\Delta X_{i}, \Delta X_{i-1}\right\rangle=0$, and the statistical properties of the movement along the path do not change with time. Therefore, the properties of the movement are fully determined by the p.d.f for $\Delta X_{i}$, which we denote as $\phi\left(\Delta X_{i}\right)$, and we consider the case for Brownian movement with zero mean,

$$
\phi\left(\Delta X_{i}\right)=\frac{1}{\xi \sqrt{2 \pi}} \exp \left(-\frac{\Delta X_{i}^{2}}{2 \xi^{2}}\right)
$$

where $\left\langle\Delta X_{i}^{2}\right\rangle=\xi^{2}$ is the variance which characterizes the mobility of the individual. The corresponding p.d.f for the step lengths $l_{i}=\left|\Delta X_{i}\right|$, is given by

$$
\phi\left(l_{i}\right)=\frac{2}{\xi \sqrt{2 \pi}} \exp \left(-\frac{l_{i}^{2}}{2 \xi^{2}}\right), \quad l_{i} \geq 0 .
$$

\footnotetext{
${ }^{1}$ The reader is redirected to Ahmed [1] for a detailed study on how variation in $\sigma$ can affect the modelling assumption which replaces the PSR with an adequate Gaussian profile. In fact, we find that $1 \leq \frac{\sigma}{\Delta x}<10$, suffices, where $\Delta x$ is the mesh step size for the spatial scale used in the numerical scheme.
} 
Here, the environment is isotropic and there is no advection of any kind, so that there is no drift in any preferable direction; we have assumed in (2.4) that the mean value is zero $\left\langle\Delta X_{i}\right\rangle=0$. Once parameter $\xi$ is known, equations (2.4) can be used to simulate the movement path [46]. It is somewhat important to note that the dynamics of the movement model are completely determined by $\xi^{2}$, which can be explicitly dependent on time. It is well known that for a discrete-time model of an inherently continuous insect movement the mean squared displacement is dependent on the time scale $\Delta t$,

$$
\left\langle X_{i}^{2}\right\rangle=\xi^{2}=2 D \Delta t
$$

however this can be extended to a continuous model where $\xi$ and $D$ can be time dependent and nonconstant $[26,42,46,50,71]$, the general case is discussed in $\S(3)$. To mimic the configuration discussed in $\S(2.1)$ over the domain $0<x<\eta$, we install a point trap at $x=0$, if an individual has position $X_{i}<0$ at any instant in time, then by configuration it is removed from the system and deemed to be trapped. The boundary at $x=\eta$ is impermeable through which no individuals can escape; if $X_{i}>\eta$ at any instant in time, then $X_{i}=\eta$ and the next step of this individual is determined by (2.4).

For an initial PSR, $N$ individuals are simply positioned at $X_{0}=\frac{\eta}{2}$ at time $t=0$. Note that, the individual based modelling equivalent of $(2.2)$ is described by

$$
\phi\left(X_{0}\right)=\frac{1}{\sqrt{2 \pi \sigma^{2}}} \exp \left(-\frac{\left(X_{0}-x_{0}\right)^{2}}{2 \sigma^{2}}\right),
$$

subject to the following; if the individuals are placed prior to the installation of the trap and reflective boundary (i.e over an infinite domain $-\infty<x<\infty$ ), then those individuals for $x<0$ and $x>\eta$ are removed and lost prior to the simulations. This is the same as distributing individuals over $-\infty<x<\infty$ and only maintaining those individuals in the finite domain $0<x<\eta$ after removing those individuals outside this domain. Consequently, we have $\tilde{N}<N$ number of individuals in the system. To reinstate the 'true' counts provided $\tilde{N}$ is known, we assume direct proportionality

$$
J(t) \approx \frac{N \tilde{J}(t)}{\tilde{N}} .
$$

Here, $\tilde{J}(t)$ are the trap counts obtained from the simulation study with $J(t)$ as the corrected count. Notice that as $\sigma \rightarrow 0$ we have that $\tilde{J}(t) \rightarrow J(t)$, the limiting value corresponding to the PSR. The initial conditions which are of ecological significance are either PSR, uniform or of Gaussian type described by (2.7) with correction given by $(2.8),[42,50]$.

The governing equation (2.1) and its properties are well understood for $D(t)=D=$ const $[1,14,46]$. An obvious extension to the constant diffusion case is to incorporate a linear type dependency $D(t)=$ $a+b t$, the latter case is essentially anomalous provided $b \neq 0$. In Fig. (1) we choose to plot the trap counts as opposed to the solution $u(x, t)$ since our primary mode of study and interest lies in trap count interpretation. The diffusion equation defined over the semi-infinite domain $0<x<\infty$ with initial PSR has analytical solution, see (A.8),

$$
J(\tau)=N \operatorname{erfc}\left(\frac{x_{0}}{\sqrt{4 \tau}}\right) \quad \text { with } \quad \tau=\int_{0}^{t} D\left(t^{\prime}\right) d t^{\prime}
$$

and in the case of initial uniform distribution, see (A.6),

$$
J(\tau)=2 U_{0} \sqrt{\frac{\tau}{\pi}} \quad \text { with } \quad \tau=\int_{0}^{t} D\left(t^{\prime}\right) d t^{\prime}
$$

Both analytic solutions are useful as approximations to the finite domain case for sufficient small time, [1]. Plot B illustrates identical trap counts for the MFA, IBM and the analytical description, with PSR relatively close to the trap boundary, i.e the effect of the reflective boundary at $x=\eta$ is negligible or unnoticed. In fact, when the time dependent diffusion coefficient is small in comparison to the spatial scale, we obtain almost identical trap counts. 

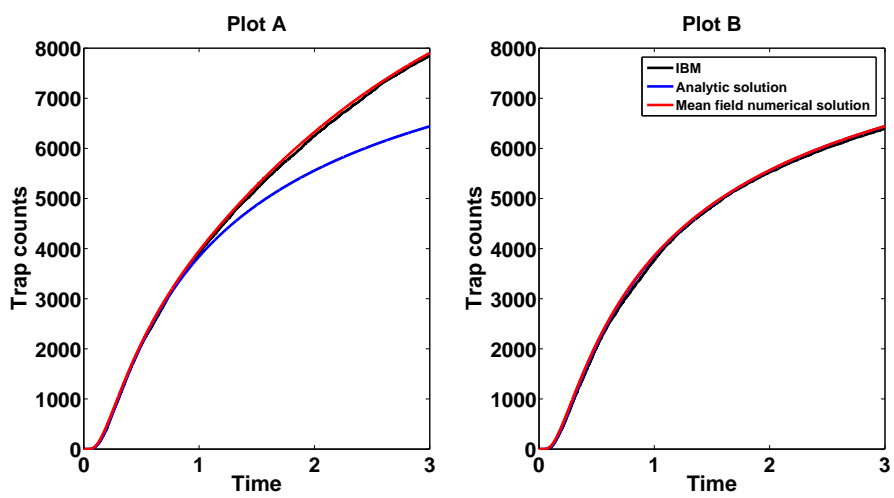

Figure 1. Plot A: Trap count comparison between the three approaches mean field numerical solution (red curve), IBM (black curve) and the analytic solution (blue curve) $J(t)$ (see (A.8)) whose movement dynamics are time dependent with initial PSR centered at $x_{0}=\frac{\eta}{2}$. Fixed parameters include; $U_{0}=N=10^{4}, \Delta t=6 \times 10^{-5}, \eta=1, x_{0}=0.5$. Individual based model with mobility parameter $\xi=\sqrt{2(a+b t) \Delta t}$ and $N=10^{4}$ individuals positioned at $X_{0}=0.5$ at time $t=0$. Mean field model with time dependent diffusion $D(t)=a+b t$ and initial population density given by (2.2). Simulation parameters $a=0.15, b=0.03$ and $\sigma=0.05$. Analytic solution given by (A.8), with $D(t)$ already described. In these plots and in all following simulations, $N$ is chosen sufficiently large so that the relative magnitude of stochastic fluctuations decreases as $N^{-\frac{1}{2}}=0.01$, a fundamental result in statistical mechanics, [2]. The magnitude of the stochastic fluctuations must be small enough for adequate comparisons with the diffusive flux. The time step $\Delta t=6 \times 10^{-5}$ (fixed for all following random walk simulations) is chosen to be sufficiently large, so that the assumption that subsequent steps are uncorrelated is feasible $[31,46]$. Plot B: Comparison between the three approaches MFA, IBM and analytic for domain length $\eta=10$ with initial PSR position centered at $x_{0}=\frac{\eta}{20}=0.5$. All initial/boundary conditions and other parameter values identical to those described in Plot A. The difference between Plot B and Plot A is the 10 fold increase in domain length, reducing the effect on the reflective boundary.

\section{Anomalous diffusion and Lévy walks}

Analogous to standard diffusion, there do exist formally equivalent approaches to anomalous diffusion [22]. The most well known approach incorporates continuous time random walks (CTRWs) into the mathematical framework, [37,57,58,62]. Kawai [32] developed a CTRW model as a counterpart to Lévy walks, however limitations appear, in particular, in terms of statistical inference. In fact, a variety of challenges arise from continuous time modelling. Often, there is an inevitable tradeoff; analytically tractable models tend to be statistically unrealistic, whilst models incorporating more statistical features are too complex. Kawai [32], focuses on Markovian Lévy process models as continuous time counterpart of Lévy walks. There are non-Markovian features such as directional persistence and autocorrelation $[7,12,63,69]$ which can also be taken into account. For example, fractional Brownian and Lévy motions are continuous-time stochastic processes with inherent non-Markovian features, examined by Reynolds [53] in their discrete time form as alternatives to Lévy walks.

A second formalism for dealing with anomalous diffusion is based on fractional time and space derivatives. The idea is based on the fact that the fractional Laplacian operator of order $\frac{\omega}{2}$ has fourier transform $-|k|^{\omega}$, consequently one can obtain any Hurst exponent in the range $0 \leq H \leq 1,[35,36]$. A comprehensive treatment of anomalous diffusion and transport can be found in Radons et al. [52]. The above formalisms are important and effective, with respect to the study of animal movement, however they 
can lead to over complications in the context of pest trapping, leaving little room for interpretation. In fact, some important questions seem to be almost forgotten: which ever pattern occurs, what are its ecological implications? How much importance should be given to the type of movement pattern in the context of a specific problem? Lévy walks are thought to be essentially different from Brownian motion but does it necessarily mean that the corresponding mathematical framework should always be different too? We aim to address these questions and elaborate on current studies where it has been shown that in the case of a genuine Lévy walk, the problem of trap count interpretation can still be addressed based on the diffusion equation $[1,50]$. Our base study, exploits the fact that the Lévy parameter $1<\mu<3$ corresponds to the super-diffusive regime [73]. The underlying issue is, what properties must be inherent in $D(t)$ so that trap counts for Lévy random walkers can be predicted, effectively. What follows in this section, is a series of test cases, leading to development and providing useful insight to address this issue.

For browsing individuals whose movement mode is of a Brownian nature, the corresponding p.d.f $\phi$ is a solution to the diffusion equation

$$
\frac{\partial \phi}{\partial t}=D \frac{\partial^{2} \phi}{\partial x^{2}}
$$

with constant diffusion coefficient $D$. The solution, subject to the initial condition $\phi\left(x-x_{0}, t=0\right)=$ $\delta\left(x-x_{0}\right)$, which means that the walker is at $x=x_{0}$ at time $t=0$, is

$$
\phi\left(x-x_{0}, t\right)=\frac{1}{\sqrt{4 \pi D t}} \exp \left(-\frac{\left(x-x_{0}\right)^{2}}{4 D t}\right) .
$$

The mean squared displacement ${ }^{2}$ (MSD) can be computed as $\left\langle X_{t}^{2}\right\rangle=2 D t$, which grows linearly with time. This property is widely regarded as a 'finger-print' of Brownian motion $[12,46,50,71,73]$. More generally, if $D=D(t)$ is explicitly dependent on time, then (3.1) can be transformed by introducing $\tau=\int_{0}^{t} D\left(t^{\prime}\right) d t^{\prime}$, see (A.2). It follows that,

$$
\left\langle X_{t}^{2}\right\rangle=2 \tau=2 \int_{0}^{t} D\left(t^{\prime}\right) d t^{\prime}
$$

For anomalous diffusive processes the MSD is not linear in time but instead has some other power law relationship given by

$$
\left\langle X_{t}^{2}\right\rangle \sim t^{2 H}, \quad H \neq \frac{1}{2}
$$

where $H$ is known as the Hurst exponent. A full comprehensive summary of movement properties with reference to $H$ is provided below, also see $[12,73]$.

(I) $H=0$, Localized and confined random walkers have vanishing $H$, [73].

(II) $0<H<\frac{1}{2}$, This case is known as sub-diffusion since the MSD increases at a slower rate than in the case of standard diffusion. This case typically occurs when waiting times are incorporated between steps [74], or if the spatial domain is constrained i.e presence of a barrier [13].

(III) $H=\frac{1}{2}$, This is the standard relation between MSD and time for diffusive movement i.e $\left\langle X_{t}^{2}\right\rangle=2 D t$, with constant diffusion coefficient $D$.

(IV) $\frac{1}{2}<H<1$, This case corresponds to a super diffusive process (known as super-diffusion) since the MSD increases at a faster rate than in the case of standard diffusion. This typically occurs when the step lengths are drawn from a distribution with infinite variance.

(V) $H=1$, The movement process is ballistic or wavelike. The MSD grows quadratically with time and this corresponds to the absolute displacement increasing linearly with time, a standard property of a wave process.

\footnotetext{
${ }^{2}$ The subcript $t$ is used in $\left\langle X_{t}^{2}\right\rangle$ to emphasise that the MSD is a time dependent statistic.
} 
(VI) $H>1$, Does not correspond to anything meaningful in the context of insect foraging. From a physical perspective, this case corresponds to accelerated motion, applicable to turbulent flows [5]. Turbulence is now understood as a multi-scale phenomenon, by large owing to the pioneering paper by Richardson [56].

More generally, complex dependencies of the MSD can appear which do not necessarily have to be of the form described by (3.4). A typical well known example is that of logarithmic ultra-slow diffusion given by $\left\langle X_{t}^{2}\right\rangle \sim t^{\gamma} \log ^{\beta}(t)$, in particular known as Sinai diffusion for $\gamma=0, \beta=4,[6]$.

\subsection{Cauchy type random walk}

A genuine Lévy walk arises if the p.d.f for the step lengths $l$ behaves at large $l$ as $\phi(l) \sim l^{-\mu},(1.1)$, with $\mu<3$. Such stochastic processes have a fat tail with undefined variance, as opposed to the thinner tails inherent in Gaussian profiles. In mathematical terms, a higher frequency of large steps means a lower rate of probability density decay at large distances. Notice however, a p.d.f with a 'fat' tail is not sufficient for identifying a Lévy walk. The central limit theorem [26] states that the sum of independent and identical distributions, with first three moments converges to a normal distribution when the number of steps tend to infinity. Viswanathan et al. [72,73] demonstrated that $\mu=2$ is the optimal parameter for a random search in any dimension. This justifies our choice to characterize the step lengths according to an inverse square law. Our study is therefore confined to trap count approximations for insect species which undergo an optimum searching strategy. Although there are a class of p.d.f's which can be considered with this parameter, they are all asymptotically characterized with step length distributions decaying according to $\sim \frac{1}{l^{2}}$, a typical example of interest is the Cauchy distribution, [26]. The corresponding p.d.f centered at $\Delta X=0$ reads,

$$
\phi(\Delta X)=\frac{k}{\pi\left(k^{2}+\Delta X^{2}\right)} .
$$

This distribution is strictly stable, has undefined moments and the central limit theorem fails. Here, $k$ is the parameter of interest. Larger $k$ implies that individuals have a greater probability of executing larger steps. A biological consequence of this is such that an individual has a greater trap hitting probability i.e greater chance of being trapped. Therefore, we expect faster convergence of the accumulated trap counts to the maximal $N$ in a bounded domain with finite $N$.

For brevity, we denote $J_{A}$ for trap counts obtained from a system whose movement dynamics are governed by a time dependent diffusive process. Correspondingly, $J_{C}$ denotes trap counts formulated from Cauchy type random walkers with step distribution $\phi(\Delta X)=\frac{k}{\pi\left(k^{2}+\Delta X^{2}\right)}$, with varying $k$. Fig. (2) depicts a comparison between $J_{A}$ with $D(t)=a+b t$ against $J_{C}$ with varying $k$. The idea is to compare counts for a suitable parameter $k$ for fixed pair $(a, b)$. Any rigorous justification is omitted, for our current purposes a qualitative observation on patterns and growth rates is sufficient. The trap count profiles are inherently different for small and large time. For small time, $J_{C}$ depicts larger trap counts (greater flux rate) in comparison to $J_{A}$, at least for those values of $k$ which have matching relevance i.e $k \geq 4 \times 10^{-5}$. For large time, we realise that $J_{A}$ depicts larger trap counts and in particular exhibits faster flux convergence to the maximal, total population, $N$. It has been confirmed from large time simulations with larger $k$ that the maximal is indeed $N$; however not presented in this paper, see [1]. Overall a phenomena of undershoot-overshoot is observed, when comparing the proposed diffusion model to the stochastic model. Our approach is pedagogical, for a better matching we need to simulate multiple trap counts $J_{A}$ with varying $a, b$ for fixed parameter $k$.

In the following Fig. (3), Plots $\mathbf{A}$ and $\mathbf{C}$ we fix $b=0.03$ and vary $a$. For small time $0<t<0.1$ (representing $<25 \%$ of total trap counts), $a$ is responsible for effective changes in $D(t)$. In Plots $\mathbf{B}$ and $\mathbf{D}$, we fix the value of $a=0.15$ and vary $b$. For relatively larger time $0<t<1.2$ (representing $\approx$ $80 \%$ of total trap counts) we have that $b$ is the underlying parameter which is responsible for effective changes in $D(t)$. Plots A and B provide simulations for an initial distribution given by (2.7) (with corrected trap counts (2.8)) for small and large time, respectively. Plots C and D provide simulations 


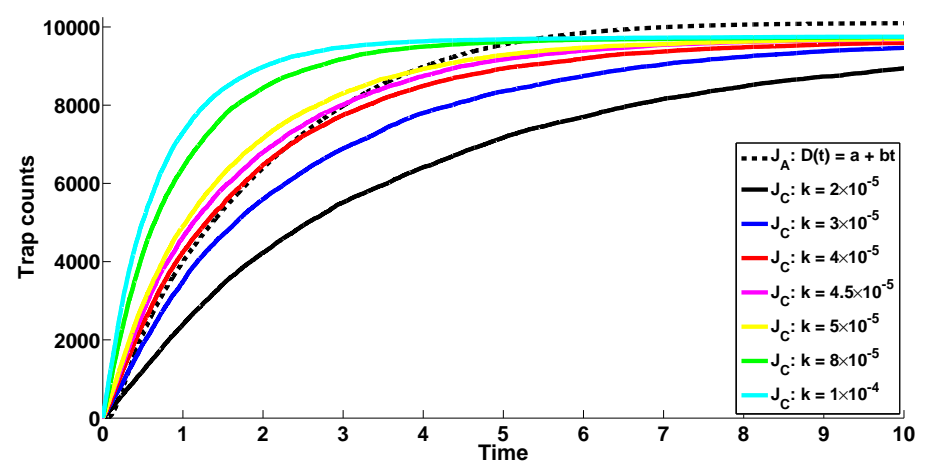

Figure 2. Fixed parameters include $N=10^{4}, \eta=1, \Delta t=6 \times 10^{-5}, \sigma=0.05$. Dashed curve: Trap counts $J_{A}$ obtained from the anomalous diffusive process with $D(t)=a+b t$, $a=0.15, b=0.03$, and initial population density of Gaussian type prescribed by $(2.2)$ with $x_{0}=\frac{\eta}{2}$. Solid realizations: Single realization trap counts $J_{C}$ for Lévy walk with step distribution $\phi(\Delta X)=\frac{k}{\pi\left(k^{2}+\Delta X^{2}\right)}$ of Cauchy type with varying $k$, and initial position of Gaussian type prescribed by (2.7) with correction given by (2.8). Notice that, $J_{C}$ converges to the maximal value $N$, at a slower rate in comparison to $J_{A}$.

Plot A
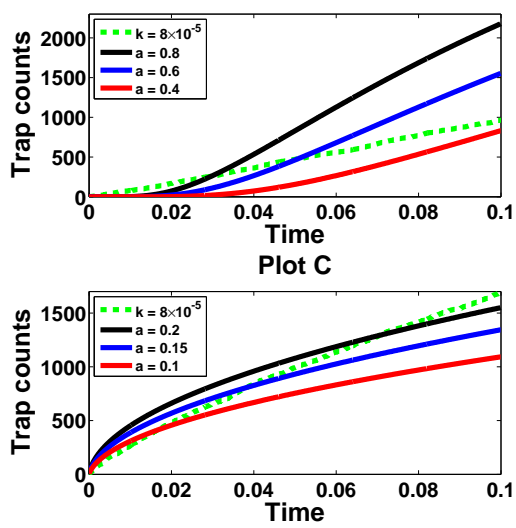

Plot B
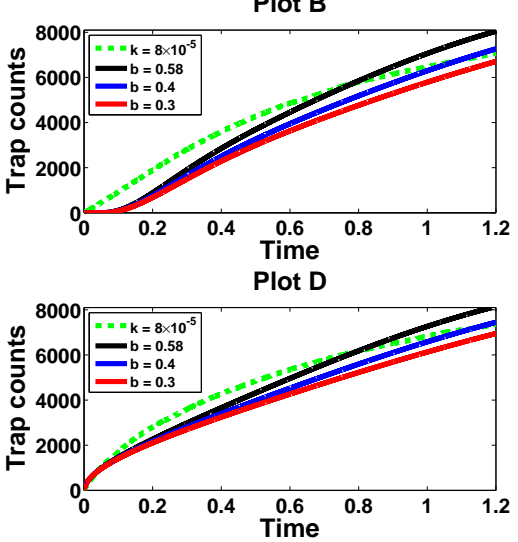

FiguRE 3. Fixed parameters include $N=10^{4}, \eta=1, \Delta t=6 \times 10^{-5}, \sigma=0.05$. Dashed realization: Single realization trap counts $J_{C}$ for Lévy walk with step distribution $\phi(\Delta X)$ of Cauchy type with fixed $k=8 \times 10^{-5}$. Plot A,B: Initial position of Gaussian type prescribed by (2.7) with correction (2.8) and $x_{0}=\frac{\eta}{2}$. Plot C,D: Initial position distributed uniformly i.e $\phi\left(X_{0}\right)=\frac{1}{\eta}$. Solid curves: Trap counts $J_{A}$ obtained from the anomalous diffusive process with $D(t)=a+b t$. Plot A,B: Initial population density of Gaussian type prescribed by (2.2) with $x_{0}=\frac{\eta}{2}$. Plot C,D: Initial uniform population density, $u(x, t=0)=U_{0}=\frac{N}{\eta}$. Plot A: $b=0.03$ with $a=0.4,0.6,0.8$. Plot B, D: $b=0.58,0.4,0.3$ with $a=0.15$. Plot C: $b=0.03$ with $a=0.1,0.15,0.2$.

for an initial uniform distribution for small and large time, respectively. We observe from Fig. (3), Plots A and B that trap count profiles $J_{A}$ undershoot for small time and overshoot for large time, an effective matching cannot be maintained. The opposite phenomena is observed in the case of initial uniform distribution, Plots C and D. Evidently, we have two conclusive messages from this section. 
Firstly, the shape of the trap count profiles depend on the initial condition, predominately for small time, i.e concavity and convexity in the case of the uniform distribution and PSR $^{3}$, respectively [1]. Secondly and more importantly, trap counts obtained from a diffusion model with linear $D(t)$ do not satisfactorily match counts from the Cauchy type Lévy walk. These patterns invoke an introduction of non-linear time dependencies. For $D(t)=a+b t$, the MSD (3.3) is

$$
\left\langle\Delta X_{t}^{2}\right\rangle=2 \int_{0}^{t} D\left(t^{\prime}\right) d t^{\prime}=2 a t+b t^{2} \sim t^{2}
$$

with Hurst exponent $H=1$. The movement process is ballistic or wavelike. We introduce the following non-linear model with generalized exponent $\nu$,

$$
D(t)=a+b t^{\nu}
$$

with analytical flux given by

$$
J(t)=\frac{2 U_{0}}{\sqrt{\pi}}\left(\int_{0}^{t}\left(a+b\left(t^{\prime}\right)^{\nu}\right) d t^{\prime}\right)^{\frac{1}{2}}=\frac{2 U_{0}}{\sqrt{\pi}}\left(a t+\frac{b t^{\nu+1}}{\nu+1}\right)^{\frac{1}{2}}, \quad \nu>-1
$$

from (A.6), on a semi-infinite domain with initial uniform density $u(x, t=0)=U_{0}$.

The MSD is computed as

$$
\left\langle\Delta X_{t}^{2}\right\rangle=2 \int_{0}^{t} D\left(t^{\prime}\right) d t^{\prime}=2 a t+\frac{2 b t^{\nu+1}}{\nu+1} \sim\left\{\begin{array}{r}
t \text { for } \nu \leq 0 \\
t^{\nu+1} \text { for } \nu>0
\end{array}\right.
$$

with Hurst exponent $H=\frac{\nu+1}{2}$ for $H>0$. The process is super diffusive provided $\frac{1}{2}<H<1$ i.e $0<\nu<1$.

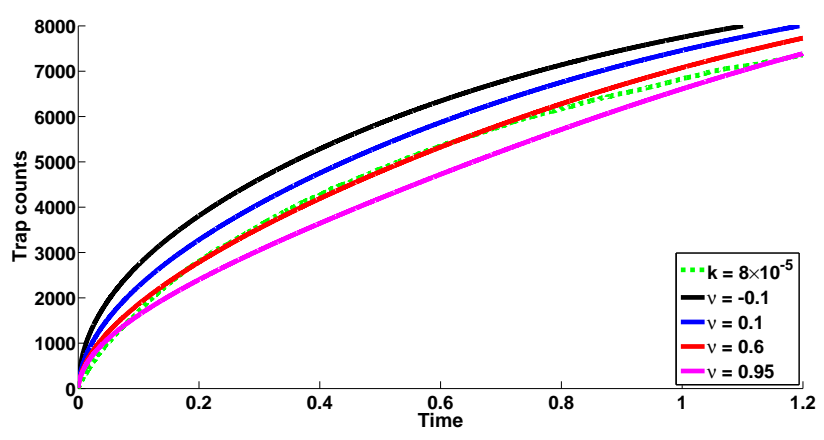

Figure 4. Fixed parameters include $N=10^{4}, \eta=1, \Delta t=6 \times 10^{-5}, \sigma=0.05$. Solid curve: Trap counts $J_{A}$ obtained from the anomalous diffusive process with $D(t)=$ $a+b t^{\nu}, a=0.2$ and $b=0.3$, with varying $\nu=0.3,0.4,0.5,0.6$. Initial uniform population density, $u(x, t=0)=U_{0}=\frac{N}{\eta}$. The process is super diffusive for $\nu=0.1,0.6,0.95$ since $\frac{1}{2}<H<1$, and sub-diffusive for $\nu=-0.1,(H=0.45)$. Dashed realizations: Single realization trap counts $J_{C}$ for Lévy walk with step distribution $\phi(\Delta X)=\frac{k}{\pi\left(k^{2}+\Delta X^{2}\right)}$ of Cauchy type with fixed $k=8 \times 10^{-5}$, and initial position distributed uniformly i.e $\phi\left(X_{0}\right)=\frac{1}{\eta}$, i.e uniformly distributed over the interval

\footnotetext{
${ }^{3}$ Recall that the initial condition $\phi\left(X_{0}\right)=\frac{1}{\sqrt{2 \pi \sigma^{2}}} \exp \left(-\frac{\left(X_{0}-x_{0}\right)^{2}}{2 \sigma^{2}}\right)$ represents a PSR in the limiting case $\sigma \rightarrow 0$. In fact $\phi\left(X_{0}\right)$ effectively models a PSR provided $\sigma$ is sufficiently small.
} 
Fig. (4) compares the trap count profiles $J_{C}$ against $J_{A}$ with $D(t)=a+b t^{\nu}$, and varying exponent $\nu$. We observe an 'improved' match for $\nu=0.6$, with smoothening of the overshoot-undershoot phenomena. In actual practice we are not interested in a detailed discussion to address the question of 'how good is the match?'. A statistical analysis is omitted at this stage, since further development is yet to follow. For our current purposes, observations on trap count patterns suffices. Apparently, diffusion models with non-linear $D(t)$ yield better trap count approximations for a system of Cauchy type random walkers.

\subsection{Parameter reduction}

The proposed non-linear dependency $D(t)=a+b t^{\nu}$ is confined to three parameters $(a, b, \nu)$. The matching process heavily depends on parameter variation, with special importance given to the exponent $\nu$. The number of parameters can be reduced by approximating the flux rate in the limit $t \rightarrow 0$. Denote $N_{i}$ as the number of individuals trapped after $i$ time steps, i.e at time $t=i \Delta t . N_{i}$ is in fact stochastic depending on the parameters inherent in the step distribution $\phi(\Delta X)$. Intuitively, the approximation reads

$$
\lim _{t \rightarrow 0} \frac{d J(t)}{d t} \approx \frac{\mathbb{E}\left(N_{i=1}\right)}{\Delta t} .
$$

Let $u_{i-1}(x)$ be the population density over space after $(i-1)$ steps of the random walk i.e at time $t=i \Delta t$. Then, for any probability density distribution $\phi(\Delta X)$ of the step $\Delta X$, the expected number $N_{i}$ of insects trapped as a result of the next $i^{\text {th }}$ step has integral representation,

$$
\mathbb{E}\left(N_{i}\right)=\int_{0}^{\eta} u_{i-1}(x) d x \int_{-\infty}^{0} \phi(x-\omega) d \omega
$$

Since $u_{i}(x)$ is unknown for arbitrary $i$, it follows that an explicit analytical expression can not be computed for $\mathbb{E}\left(N_{i}\right)$, except for $i=1$, in the case the initial distribution $u_{0}(x)$ is prescribed.

$$
\mathbb{E}\left(N_{i=1}\right)=\int_{0}^{\eta} u_{0}(x) d x \int_{-\infty}^{0} \phi(x-\omega) d \omega
$$

For an initial uniform density $u_{0}(x)=U_{0}$ with Cauchy step distribution $\phi$,

$$
\mathbb{E}\left(N_{i=1}\right)=\int_{0}^{\eta} U_{0} d x \int_{-\infty}^{0} \frac{k}{\pi\left(k^{2}+(x-\omega)^{2}\right)} d \omega
$$

and on evaluation,

$$
\mathbb{E}\left(N_{i=1}\right)=\frac{U_{0} \eta}{2}-\frac{U_{0} k}{\pi}\left(\frac{\eta}{k} \arctan \left(\frac{\eta}{k}\right)-\frac{1}{2} \ln \left(1+\left(\frac{\eta}{k}\right)^{2}\right)\right)=U_{0} \Psi(\eta, k) .
$$

We introduce $\Psi(\eta, k)$ as an alternative notation to emphasise the dependencies on domain length $\eta$ and Cauchy parameter $k$. Alternatively, the flux rate can be obtained from the solution of the diffusion equation over the semi-infinite domain, assuming that domain length $\eta$ is large enough, so that the effect of domain finiteness can be neglected after one time step $\Delta t$.

Equation (A.6) states

$$
J(t)=\frac{2 U_{0}}{\sqrt{\pi}}\left(\int_{0}^{t} D\left(t^{\prime}\right) d t^{\prime}\right)^{\frac{1}{2}}
$$

and on differentiating with respect to $t(3.9)$ reads,

$$
\lim _{t \rightarrow 0} \frac{d J(t)}{d t}=\lim _{t \rightarrow 0} \frac{U_{0} D(t)}{\sqrt{\pi}\left(\int_{0}^{t} D\left(t^{\prime}\right) d t^{\prime}\right)^{\frac{1}{2}}} \approx \frac{\mathbb{E}\left(N_{i=1}\right)}{\Delta t} .
$$


For the proposed structure $D(t)=a+b t^{\nu}$ the approximation (3.13) fails, since the limiting value is not finite. Consequently, parameter reduction using this methodology is limited. However, to demonstrate the effectiveness of such a relation, consider $D(t)=a t+b t^{\nu}$ as a test case. The MSD is

$$
\left\langle\Delta X_{t}^{2}\right\rangle=2 \int_{0}^{t} D\left(t^{\prime}\right) d t^{\prime}=a t^{2}+\frac{2 b t^{\nu+1}}{\nu+1} \sim\left\{\begin{array}{r}
t^{2} \text { for } \nu \leq 1 \\
t^{\nu+1} \text { for } \nu>1
\end{array}\right.
$$

with Hurst exponent $H=\frac{\nu+1}{2}$ provided $\nu>1$. We find that in the limiting case for $\nu>1$,

$$
\lim _{t \rightarrow 0} \frac{d J(t)}{d t}=\lim _{t \rightarrow 0} \frac{U_{0}}{\sqrt{\pi}} \frac{a+b t^{\nu-1}}{\left(\frac{1}{2} a+\frac{b t^{\nu}}{\nu+1}\right)^{\frac{1}{2}}}=U_{0} \sqrt{\frac{2 a}{\pi}},
$$

and using (3.13) it follows that,

$$
a \approx \frac{\pi}{2}\left(\frac{\mathbb{E}\left(N_{i=1}\right)}{U_{0} \Delta t}\right)^{2}=\frac{\pi}{2}\left(\frac{\Psi(\eta, k)}{\Delta t}\right)^{2} .
$$

Notice that this methodology works for diffusion coefficients whose limiting value defined by (3.13) exists and is finite. There are in fact a class of non-linear type dependencies for which this methodology can be used to reduce parameters.

Alternatively, the number of insects caught after one time step can be explicitly found from the diffusion model, leading to the approximation $J(\Delta t) \approx \mathbb{E}\left(N_{i=1}\right)$. From (A.6) we find that

$$
J(\Delta t)=\frac{2 U_{0}}{\sqrt{\pi}}\left(\int_{0}^{\Delta t} D\left(t^{\prime}\right) d t^{\prime}\right)^{\frac{1}{2}} \approx \mathbb{E}\left(N_{i=1}\right) .
$$

On substituting the test case $D(t)=a t+b t^{\nu}$ into (3.16), using (3.12) and rearranging for $a$, we obtain

$$
a \approx \frac{\pi}{2}\left(\frac{\Psi(\eta, k)}{\Delta t}\right)^{2}-\frac{2 b}{(1+\nu)(\Delta t)^{1-\nu}} .
$$

Here (3.17) forms a stronger approximation, in the sense that the methodology can be used for a wider class of diffusion coefficients, such as $D(t)=a+b t^{\nu}$, whereas (3.13) failed. Note however, (3.15) is totally independent of other parameters in $D(t)$, namely $b, \nu$ and therefore can be more useful in some instances.

\section{Introducing complex time dependencies}

Patterns inherent in the trap count profiles (see Fig. (4)) suggest that the flux rate $\frac{d J(t)}{d t}$ decreases with time i.e the rate at which individuals are trapped decreases. Correspondingly, we propose a generalized diffusion coefficient which grows and decays for small/large time respectively,

$$
D(t)=\sum_{j=0}^{M} \alpha_{j} t^{\beta_{j}} \exp \left(-\gamma_{j} t^{\delta_{j}}\right)
$$

where $\alpha_{j}, \beta_{j}, \gamma_{j}$ and $\delta_{j}$ are positive for all $j$. The model (4.1) has complex structure and it makes sense to use the first two leading terms in the expansion,

$$
D(t)=\alpha_{0} t^{\beta_{0}} \exp \left(-\gamma_{0} t^{\delta_{0}}\right)+\alpha_{1} t^{\beta_{1}} \exp \left(-\gamma_{1} t^{\delta_{1}}\right) .
$$

Large time asymptotic behaviour is governed by $\gamma_{j}, \delta_{j}$. Relevant simplifications include $\gamma_{0}=\gamma_{1}=\gamma$, so that different decay rates in (4.2) are determined purely by exponents $\delta_{0}, \delta_{1}$. For small time, $D(t) \approx \alpha_{0} t^{\beta_{0}}$ 
provided $\beta_{1}>\beta_{0}$, therefore set $\beta_{0}=1$ so that the leading term behaves linearly, subject to exponential decay. Here, $\beta_{1}>1$ is a 'free' exponent, designed to keep the second term as a 'correction' term controlling deviations from linearity. It follows that,

$$
D(t)=\alpha_{0} t \exp \left(-\gamma t^{\delta_{0}}\right)+\alpha_{1} t^{\beta_{1}} \exp \left(-\gamma t^{\delta_{1}}\right) .
$$

In accordance with (A.6), for homogenously distributed individuals at $t=0$, it would be advantageous if (4.3) is written in an integral form, intuitively we propose the following

$$
\int_{0}^{t} D\left(t^{\prime}\right) d t^{\prime}=\frac{1}{2} \tilde{\alpha}_{0} t^{2} \exp \left(-\tilde{\gamma} t^{\tilde{\delta}_{0}}\right)+\tilde{\alpha}_{1} t^{\tilde{\beta}} \exp \left(-\tilde{\gamma} t^{\tilde{\delta}_{1}}\right)
$$

with analytical flux

$$
J(t)=\frac{2 U_{0}}{\sqrt{\pi}}\left(\frac{1}{2} \tilde{\alpha}_{0} t^{2} \exp \left(-\tilde{\gamma} \tilde{t}^{\tilde{\delta}_{0}}\right)+\tilde{\alpha}_{1} t^{\tilde{\beta}} \exp \left(-\tilde{\gamma} t^{\tilde{\delta}_{1}}\right)\right)^{\frac{1}{2}}
$$

where $\tilde{\alpha}_{0}, \tilde{\alpha}_{1}, \tilde{\beta}, \tilde{\gamma}, \tilde{\delta}_{0}$ and $\tilde{\delta}_{1}$ are new redefined parameters. The flux rate in the limit $t \rightarrow 0$ can be computed as,

$$
\begin{gathered}
\lim _{t \rightarrow 0} \frac{d J}{d t}=\frac{U_{0}}{\sqrt{\pi}} \lim _{t \rightarrow 0} \frac{\tilde{\alpha}_{0}\left(1-\frac{1}{2} \tilde{\gamma} \tilde{\delta}_{0} t^{\tilde{\delta}_{0}}\right) \exp \left(-\tilde{\gamma} t^{\tilde{\delta}_{0}}\right)+\tilde{\alpha}_{1} t^{\tilde{\beta}-2}\left(\tilde{\beta}-\tilde{\gamma} \tilde{\delta}_{1} t^{\tilde{\delta}_{1}}\right) \exp \left(-\tilde{\gamma} t^{\tilde{\delta}_{1}}\right)}{\left(\frac{1}{2} \tilde{\alpha}_{0} \exp \left(-\tilde{\gamma} t^{\tilde{\delta}_{0}}\right)+\tilde{\alpha}_{1} t^{\tilde{\beta}-2} \exp \left(-\tilde{\gamma} t^{\tilde{\delta}_{1}}\right)\right)^{\frac{1}{2}}} \\
=U_{0} \sqrt{\frac{2 \tilde{\alpha}_{0}}{\pi}}
\end{gathered}
$$

provided $\tilde{\beta}>2$ and $\tilde{\gamma}, \tilde{\delta}_{0}, \tilde{\delta}_{1}>0$. From (3.13), approximation for $\tilde{\alpha}_{0}$ in model (4.4) is in accordance with (3.15),

$$
\tilde{\alpha}_{0} \approx \frac{\pi}{2}\left(\frac{\mathbb{E}\left(N_{i=1}\right)}{U_{0} \Delta t}\right)^{2}=\frac{\pi}{2}\left(\frac{\Psi(\eta, k)}{\Delta t}\right)^{2} .
$$

Notice that $\lim _{t \rightarrow \infty} J(t)=0$ for model (4.5) provided $\tilde{\gamma}, \tilde{\delta}_{0}, \tilde{\delta}_{1}>0$. For a system with no migration properties we expect the total population to be conserved, i.e $\lim _{t \rightarrow \infty} J(t)=U_{0} \eta=N$. Therefore the model fails in this limiting value and is only applicable for time with validity period $t<t_{\max }$, found from simulations. However, most insect monitoring studies are concerned with short time dynamics, particularly in the context of trapping. The following three cases classify possible scenarios with reference to $D(t)$.

(I) If $\lim _{t \rightarrow \infty} D(t)=\infty$ then $J(t)>N$ for some $t$ and it follows that $J\left(t_{\max }\right)=N$.

(II) If $\lim _{t \rightarrow \infty} D(t)=-\infty$ and $J(t)<N$ for all $t$, we obtain $t_{\max }$ from the flux rate $\frac{d J\left(t_{\max }\right)}{d t}=0$ or alternatively the first zero of $D\left(t_{\max }\right)=0$. However, if $J(t) \geq N$ for some $t$ then $J(t)=N$ with $t_{\max }=\min (\hat{t})$, since $\hat{t}$ is multi-valued.

(III) If $\lim _{t \rightarrow \infty} D(t)=$ const $\geq 0$ and $J(t) \geq N$ for some $t$ then $J\left(t_{\max }\right)=N$. However, if $J(t)<N$ for all $t$ then the validity period is questionable.

In Fig. (5), the black curve depicts trap counts for the super diffusive process with $D(t)=a+b t^{\nu}$ with $a=0.2, b=0.3, \nu=0.6$, developed in $\S(3.1)$ see Fig. (4). The blue curve represents the analytic solution for diffusion coefficient $D(t)=a+b t^{\nu}$ given by $(3.7)$,

$$
J(t)=\frac{2 U_{0}}{\sqrt{\pi}}\left(a t+\frac{b t^{\nu+1}}{\nu+1}\right)^{\frac{1}{2}}, \quad \nu>-1, \quad t<t_{\max },
$$




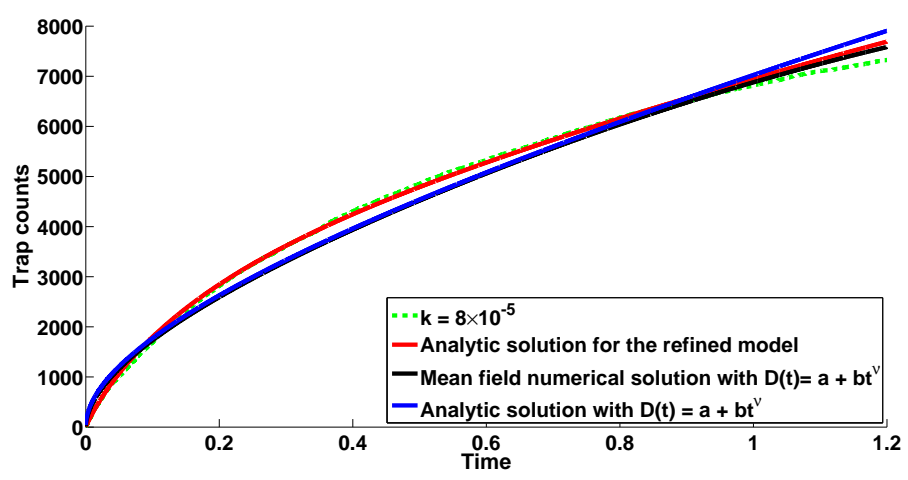

FiguRE 5. Comparison of trap count profiles obtained from the system with Cauchy type random walkers $\left(J_{C}\right)$ against time dependent diffusive flux $\left(J_{A}\right)$. Fixed parameters include $N=U_{0}=10^{4}, \eta=1, \Delta t=6 \times 10^{-5}$. Analytic solutions are given over the semi-infinite domain $x>0$. The refined model for the diffusion coefficient with structure $\int_{0}^{t} D\left(t^{\prime}\right) d t^{\prime}=\frac{1}{2} \tilde{\alpha}_{0} t^{2} \exp \left(-\tilde{\gamma} t^{\tilde{\delta}_{0}}\right)+\tilde{\alpha}_{1} t^{\tilde{\beta}} \exp \left(-\tilde{\gamma} t^{\tilde{\delta}_{1}}\right)$ (see $\left.(4.5)\right)$ provides an improved trap count matching as opposed to the super diffusive process $D(t)=a+b t^{\nu}, 0<\nu<$ 1, (3.7). Green dashed realization: Trap counts $J_{C}$ for movement process with Cauchy distributed steps $\phi(\Delta X)$ (see (3.5)) and parameter $k=8 \times 10^{-5}$. Initial position distributed uniformly i.e $\phi\left(X_{0}\right)=\frac{1}{\eta}$. Red solid curve: Analytic solution $J_{A}$ given by (4.5), with parameters $\tilde{\alpha}_{0} \approx \frac{\pi}{2}\left(\frac{\mathbb{E}\left(N_{1}\right)}{U_{0} \Delta t}\right)^{2}=30.8012, \tilde{\gamma}=4, \tilde{\delta}_{0}=0.3, \tilde{\delta}_{1}=0.4$, $\tilde{\alpha}_{1}=5, \tilde{\beta}=3$. Model only valid for time $t<t_{\max }=2.83$, here $J\left(t_{\max }\right)=N$ with $\lim _{t \rightarrow \infty} D(t)=$ const $\geq 0$. Black solid curve: Mean field approach with trap counts obtained from the numerical solution (via implicit finite difference scheme) over the domain $0<x<\eta$ with diffusion coefficient $D(t)=a+b t^{\nu}$, and parameters $a=0.2$, $b=0.3$ and $\nu=0.6$ and initial population density $u(x, t=0)=U_{0}=\frac{N}{\eta}$. Blue solid curve: Analytic solution given by (3.7), with parameters $a=0.2, b=0.3$ and $\nu=0.6$. Model only valid for time $t<t_{\max }=1.71$, here $J\left(t_{\max }\right)=N$ with $\lim _{t \rightarrow \infty} D(t)=\infty$.

with validity period $t<t_{\max }=1.71$. Notice here that (3.6) is on the order of the spatial scale for large time, the reflective boundary has an effect on trap counts and therefore we expect a discrepancy. The green dashed realization represents the trap counts simulated from a system with uniform initial position, whose movement dynamics are governed by Cauchy distributed steps. Conclusively, Fig. (5) illustrates the improvement in matching with the introduction of $D(t)$ proposed in integral form by (4.4),

$$
\int_{0}^{t} D\left(t^{\prime}\right) d t^{\prime}=\frac{1}{2} \tilde{\alpha}_{0} t^{2} \exp \left(-\tilde{\gamma} t^{\tilde{\delta}_{0}}\right)+\tilde{\alpha}_{1} t^{\tilde{\beta}} \exp \left(-\tilde{\gamma} t^{\tilde{\delta}_{1}}\right), \quad t<t_{\max }
$$

The leading coefficient $\tilde{\alpha}_{0} \approx \frac{\pi}{2}\left(\frac{\mathbb{E}\left(N_{i=1}\right)}{U_{0} \Delta t}\right)^{2}=30.8012$ is approximated using the flux gradient approximation after one time step, see (3.15). Further simplification of (4.4) can be introduced by setting $\tilde{\delta}_{0}=\tilde{\delta}_{1}=\hat{\delta}$

$$
\int_{0}^{t} D\left(t^{\prime}\right) d t^{\prime}=\left(\frac{1}{2} \hat{\alpha}_{0} t^{2}+\hat{\alpha}_{1} t^{\hat{\beta}}\right) \exp \left(-\hat{\gamma} t^{\hat{\delta}}\right), \quad t<t_{\max }
$$

with analytical flux

$$
J(t)=\frac{2 U_{0}}{\sqrt{\pi}}\left(\left(\frac{1}{2} \hat{\alpha}_{0} t^{2}+\hat{\alpha}_{1} t^{\hat{\beta}}\right) \exp \left(-\hat{\gamma} t^{\hat{\delta}}\right)\right)^{\frac{1}{2}}, \quad t<t_{\max },
$$


and flux rate in the limit $t \rightarrow 0$,

$$
\lim _{t \rightarrow 0} \frac{d J}{d t}=\frac{U_{0}}{\sqrt{\pi}} \lim _{t \rightarrow 0} \frac{\left(\hat{\alpha}_{0}\left(1-\frac{1}{2} \hat{\gamma} \hat{\delta} t^{\hat{\delta}}\right)+\hat{\alpha}_{1} t^{\hat{\beta}-2}\left(\hat{\beta}-\hat{\gamma} \hat{\delta} t^{\hat{\delta}}\right)\right)}{\left(\frac{1}{2} \hat{\alpha}_{0}+\hat{\alpha}_{1} t^{\hat{\beta}-2}\right)^{\frac{1}{2}}} \exp \left(-\frac{\hat{\gamma}}{2} t^{\hat{\delta}}\right)=U_{0} \sqrt{\frac{2 \hat{\alpha}_{0}}{\pi}} .
$$

Provided $\hat{\beta}>2$ and $\hat{\gamma}, \hat{\delta}>0$, an approximation for $\hat{\alpha}_{0}$ is in accordance with (3.15),

$$
\hat{\alpha}_{0} \approx \frac{\pi}{2}\left(\frac{\mathbb{E}\left(N_{i=1}\right)}{U_{0} \Delta t}\right)^{2}=\frac{\pi}{2}\left(\frac{\Psi(\eta, k)}{\Delta t}\right)^{2} .
$$

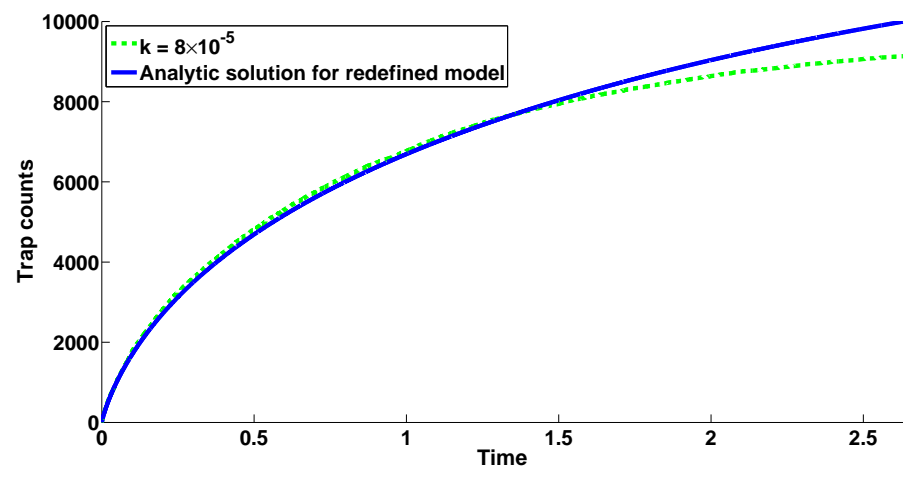

Figure 6. Comparison of trap count profiles obtained from the system with Cauchy type random walkers $\left(J_{C}\right)$ against time dependent diffusive flux $\left(J_{A}\right)$. Fixed parameters include $N=U_{0}=10^{4}, \eta=1, \Delta t=6 \times 10^{-5}$. Analytic solutions are given over the semi-infinite domain $x>0$. Green dashed realization: Trap counts $J_{C}$ for movement process with Cauchy distributed steps $\phi(\Delta X)$ (see (3.5)) and parameter $k=8 \times 10^{-5}$. Initial position distributed uniformly i.e $\phi\left(X_{0}\right)=\frac{1}{\eta}$. Blue solid curve: Analytic solution $J_{A}$ given by given by $J(t)=\frac{2 U_{0}}{\sqrt{\pi}}\left(\left(\frac{1}{2} \hat{\alpha}_{0} t^{2}+\hat{\alpha}_{1} t^{\hat{\beta}}\right) \exp \left(-\hat{\gamma} t^{\hat{\delta}}\right)\right)^{\frac{1}{2}}$, see (4.7), with parameters $\hat{\alpha}_{0}=\frac{\pi}{2}\left(\frac{\mathbb{E}\left(N_{1}\right)}{U_{0} \Delta t}\right)^{2}=30.8012, \hat{\gamma}=4.07, \hat{\delta}=0.32, \hat{\alpha}_{1}=5.2, \hat{\beta}=3$. Model only valid for time $t<t_{\max }=2.63$, here $J\left(t_{\max }\right)=N$ with $\lim _{t \rightarrow \infty} D(t)=$ const $\geq 0$.

For parameters $\hat{\alpha}_{0}=30.8012, \hat{\gamma}=4.07, \hat{\delta}=0.32, \hat{\alpha}_{1}=5.2, \hat{\beta}=3$ with $\hat{\alpha}_{0}$ defined through (3.15), we find that $D(t)>0$ for sufficient time (see Fig. (7)) over which at least $70 \%$ of total individuals are trapped, here large time simulations are omitted since interest lies in short time dynamics. Conclusively, for this parameter choice the model (4.7) is successful in predicting at least $70 \%$ of total trap counts. 

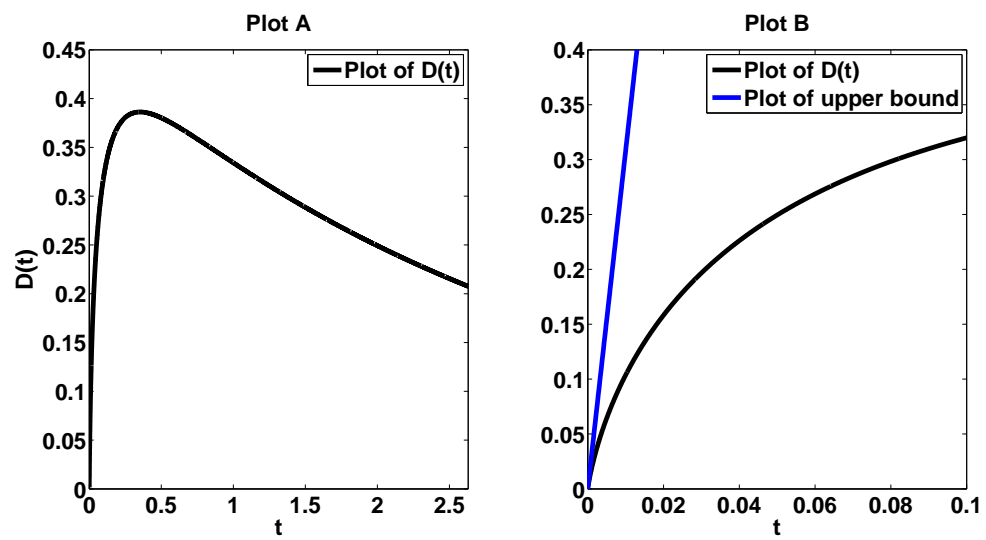

Figure 7. Plot A: Plot of the diffusion coefficient $D(t)$ proposed by the model $\int_{0}^{t} D\left(t^{\prime}\right) d t^{\prime}=\left(\frac{1}{2} \hat{\alpha}_{0} t^{2}+\hat{\alpha}_{1} t^{\hat{\beta}}\right) \exp \left(-\hat{\gamma} t^{\hat{\delta}}\right)$ described by (4.6) for $t<t_{\max }=2.63$, with set parameters $\hat{\alpha}_{0}=30.8012, \hat{\gamma}=4.07, \hat{\delta}=0.32, \hat{\alpha}_{1}=5.2, \hat{\beta}=3$, identical to those in Fig. (6). Plot B: The blue curve is a plot of the upper bound $D(t) \leq \hat{\alpha}_{0} t$, for a derivation of the upper bound see [1]. The black curve is the plot of the diffusion coefficient seen in Plot $\mathbf{A}$ over time $0<t<0.1$, for comparative purposes.

\subsection{Non-linear regressional analysis}

The following questions remain: Is the diffusive approximation suitable for other values of $k$ ? How good is the fit and can it be statistically quantified? The choice of statistical tools are broad, the recent trend is to calculate the maximum likelihood function and Akaike weights $[8,23]$ as they are regarded more reliable than other approaches amongst the literature [50]. However, to avoid any unnecessary complexity, the latter question can be addressed using non-linear regressional techniques [17]. To obtain a data set, we generate a single realization for some $k$. The time $t=T$ can be obtained from simulations for a typical count $J_{C}=m N$, where $m$, is the percentage of total trap counts. The interval is then partitioned into $p$ parts $t_{i}=\frac{i T}{p}$ for $i=1,2, \ldots, p$ with respective counts $J_{C}\left(t_{i}\right)$. Here, $T$ is the trap exposure until $m \%$ of the population is trapped. A second realization is generated with the same parameter $k$, and another set of counts are obtained at the same discrete times. Repeating in this manner, we can collect $r$ samples and compute the average to reduce the magnitude of stochastic fluctuations. Denote $J_{C}^{r}\left(t_{i}\right)$ as the trap counts (for a system of Cauchy random walkers) at discrete times $t_{i}$ for the $r^{\text {th }}$ realization. The data set

$$
\left(t_{i},\left\langle J_{C}^{r}\left(t_{i}\right)\right\rangle\right)=\left(t_{i}=\frac{i T}{p}, \frac{1}{r} \sum_{q=1}^{r} J_{C}^{q}\left(\frac{i T}{p}\right)\right)
$$

depicts $p$ points along the trap count profile. We use the statistical package NLREG ${ }^{4}$ and propose (4.7) as a candidate for parameter estimation. For detailed tabulated values for $t_{i}$ and $J_{C}^{r}$ (see Appendix B Tables 1,2 for $k=5 \times 10^{-5}, 8 \times 10^{-5}$, respectively).

Corresponding to Fig. (8), the table below summarises important statistics and measures for trap counts $\left(t_{i},\left\langle J_{C}^{r}\left(t_{i}\right)\right\rangle\right), i=1,2, \ldots, p=20$, (see (4.8)) and those counts predicted by the diffusion model (4.7). Here, $\vartheta$ is tabulated solely for the interest of the reader. Commenting on the remaining measures suffices, and allows us to address the question 'how well does the diffusion model fit?' from a quantitative perspective. Relative to the total population $N=10^{4}$ the measures $\sum d,\langle d\rangle, d_{\max }, \sum d^{2}$ and $E$ are very small, indicating that the diffusion model is successful in predicting trap counts, with better fitting for

\footnotetext{
${ }^{4}$ NLREG (C)Phillip H. Sherrod; a demonstration version is available from http://www.nlreg.com and the algorithm description can be found in Dennis et al. [17].
} 


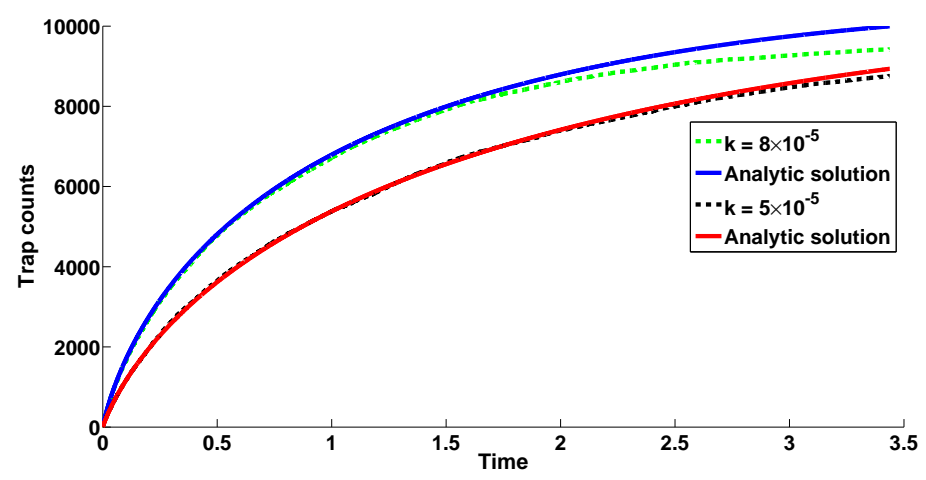

FiguRE 8. Comparison of trap count profiles obtained from the system with Cauchy type random walkers $\left(J_{C}\right)$ against time dependent diffusive flux $\left(J_{A}\right)$. Fixed parameters include $N=U_{0}=10^{4}, \eta=1, \Delta t=6 \times 10^{-5}$. Analytic solutions are given over the semi-infinite domain $x>0$. Black/Green dashed realization: Trap counts $J_{C}$ for movement process with Cauchy distributed steps $\phi(\Delta X)$ (see (3.5)), with parameters $k=5 \times 10^{-5}, 8 \times 10^{-5}$, respectively. Initial position distributed uniformly i.e $\phi\left(X_{0}\right)=\frac{1}{\eta}$. Red solid curve: Analytic solution $J_{A}$ given by given by $J(t)=\frac{2 U_{0}}{\sqrt{\pi}}\left(\left(\frac{1}{2} \hat{\alpha}_{0} t^{2}+\hat{\alpha}_{1} t^{\hat{\beta}}\right) \exp \left(-\hat{\gamma} t^{\hat{\delta}}\right)\right)^{\frac{1}{2}}$, see $(4.7)$, with best fit parameter estimates via non-linear regression $\hat{\alpha}_{0}=4.8805, \hat{\alpha}_{1}=11.4265, \hat{\beta}=2.2438, \hat{\gamma}=4.1108, \hat{\delta}=0.2816$. See Appendix B Table (1). Validity period for diffusion model $t<t_{\max }=5.65$, here $J\left(t_{\max }\right)=N$ with $\lim _{t \rightarrow \infty} D(t)=$ const $\geq 0$. Blue solid curve: Analytic solution $J_{A}$ given by given by $J(t)=\frac{2 U_{0}}{\sqrt{\pi}}\left(\left(\frac{1}{2} \hat{\alpha}_{0} t^{2}+\hat{\alpha}_{1} t^{\hat{\beta}}\right) \exp \left(-\hat{\gamma} t^{\hat{\delta}}\right)\right)^{\frac{1}{2}}$, see (4.7), with best fit parameter estimates via non-linear regression $\hat{\alpha}_{0}=22.1201, \hat{\alpha}_{1}=27.2169, \hat{\beta}=2.2949$, $\hat{\gamma}=4.6597, \hat{\delta}=0.2854$. See Appendix B Table (2). Validity period for diffusion model $t<t_{\max }=3.44$, here $J\left(t_{\max }\right)=N$ with $\lim _{t \rightarrow \infty} D(t)=$ const $\geq 0$.

\begin{tabular}{ccc}
\hline & $k=5 \times 10^{-5}$ & $8 \times 10^{-5}$ \\
\hline Sum of deviations $\sum d$ & 0.2223 & 0.6752 \\
Average deviation $\langle d\rangle$ & 6.023 & 4.438 \\
Maximum deviation $d_{\text {max }}$ & 15.37 & 11.32 \\
Sum of squared deviations $\sum d^{2}$ & 1021 & 580.2 \\
Standard Error of estimate $E$ & 8.25 & 6.219 \\
Durbin Watson test for autocorrelation $\vartheta,($ see $[18])$ & 1.903 & 1.848 \\
\hline
\end{tabular}

$k=8 \times 10^{-5}$. Note that the model is fitted for $m=0.7$ i.e $70 \%$ of total trap counts. All inferences based on the measures are up to time $T$, the trap exposure until $70 \%$ of the population is trapped. Evidently, from Fig. (8), after a longer trap exposure a better fitting is realised for $k=5 \times 10^{-5}$. In actual practice, (4.7) is a better approximation for counts which slowly accumulate.

\section{Concluding remarks}

In the context of integrated pest management, in particular, trapping studies none of the cases provided in the ecological literature with evidence of a Lévy walk appear to be free of criticism. In principle, trap count interpretations can often be subjective [50]. Moreover, there are concerns as to whether concrete evidence indicating a particular movement pattern can be obtained at all due to the complexity and 
variability of the behavioural response that pests exhibit to inherently stochastic environmental factors. Standard statistical tools sometimes cannot distinguish between power law and exponential rate of decay in the step size distribution [51]. The observed movement type can depend on technical details of data collection such as the time scale of the study [3] and/or the time resolution at which the data are obtained [32]. Pooling together movement tracks of non-identical individuals (of the same species) can create the appearance of a Lévy walk [48]. In some cases, a correlated random walk can be mistaken for a Lévy walk as it results in a similar pattern [54]. Also, animals of different taxa often employ more than one movement mode [38,39]; if each of those modes is a Brownian walk, their mixture results in a composite Brownian walk that can have the appearance of a Lévy walk $[16,28]$. We reiterate those questions raised in $\S(3)$ : Which ever pattern occurs, what are the ecological implications? How much importance should be given to the type of movement pattern in the context of pest trapping and is an alternative mathematical framework necessary? In this paper, we have shown that diffusion models with complex time dependencies (4.6) provide an alternative framework for the Cauchy type random walk. In particular, the diffusive flux (4.7) successfully predicts passive trap catches for pests whose dynamics are Lévy by nature, with exponent $\mu=2$. Moreover, better predictions are obtained with the model proposed by (4.7) as opposed to anomalous diffusive processes in the super diffusive regime (3.7). Evidently, our study indicates that even in the case of a genuine Lévy walk, the problem of trap count interpretation can be addressed with a high precision based on the diffusion equation $[1,50]$.

\section{Appendices}

\section{A. Analytic solutions for the 1D diffusion equation}

Analytic solutions can be found for the spatio-temporal poulation density $u(x, t)$ over the semi-infinite domain for the governing equation,

$$
\frac{\partial u}{\partial t}=D(t) \frac{\partial^{2} u}{\partial x^{2}}, \quad 0<x<\infty, t>0
$$

with diffusion coefficient explicitly dependent on time. The prescribed boundary conditions are the zero density $u(x=0, t)=0$ and arbitrary initial population density $u(x, t=0)=\psi(x)$. By introducing a change of variable $\tau=\int_{0}^{t} D\left(t^{\prime}\right) d t^{\prime}$, (A.1) transforms to,

$$
\frac{\partial u}{\partial \tau}=\frac{\partial^{2} u}{\partial x^{2}}, \quad 0<x<\infty, \tau>0
$$

Correspondingly, the boundary conditions are $u(x=0, \tau)=0$ and $u(x, \tau=0)=\psi(x)$.

The fundamental solution [14] is

$$
u(x, \tau)=\frac{1}{\sqrt{4 \pi \tau}} \int_{0}^{\infty} \psi(\hat{x}) G(x, \hat{x}, \tau) d \hat{x}
$$

where

$$
G(x, \hat{x}, \tau)=\exp \left(-\frac{(x-\hat{x})^{2}}{4 \tau}\right)-\exp \left(-\frac{(x+\hat{x})^{2}}{4 \tau}\right)
$$

is the Green's function for the diffusion equation over the semi-infinite domain.

(I) In the case where $\psi(x)=U_{0}$ is homogeneous, (A.3) reduces to

$$
\begin{gathered}
u(x, \tau)=\frac{U_{0}}{\sqrt{4 \pi \tau}} \int_{0}^{\infty} G(x, \hat{x}, \tau) d \hat{x} \\
=\frac{U_{0}}{\sqrt{4 \pi \tau}} \int_{0}^{\infty}\left\{\exp \left(-\frac{(x-\hat{x})^{2}}{4 \tau}\right)-\exp \left(-\frac{(x+\hat{x})^{2}}{4 \tau}\right)\right\} d \hat{x}=\frac{U_{0}}{\sqrt{4 \pi \tau}} \cdot \sqrt{4 \pi \tau} \operatorname{erf}\left(\frac{x}{\sqrt{4 \tau}}\right)
\end{gathered}
$$




$$
\Rightarrow u(x, \tau)=U_{0} \operatorname{erf}\left(\frac{x}{\sqrt{4 \tau}}\right),
$$

where $\operatorname{erf}(z)$ is the standard error function defined by $\operatorname{erf}(z)=\frac{2}{\sqrt{\pi}} \int_{0}^{z} \exp \left(-z^{\prime 2}\right) d z^{\prime}$.

The total flux is computed as

$$
J(\tau)=\left.\int_{0}^{\tau} \frac{\partial u\left(x, \tau^{\prime}\right)}{\partial x}\right|_{x=0} d \tau^{\prime}=2 U_{0} \sqrt{\frac{\tau}{\pi}} \quad \text { or } \quad J(t)=\frac{2 U_{0}}{\sqrt{\pi}}\left(\int_{0}^{t} D\left(t^{\prime}\right) d t^{\prime}\right)^{\frac{1}{2}}
$$

which reduces to

$$
J(t)=2 U_{0} \sqrt{\frac{D t}{\pi}}
$$

in the constant diffusive case.

(II) In the case of a point source release (PSR) centralised about $x_{0}, u(x, t=0)=\psi(x)=N \delta\left(x-x_{0}\right)$. From (A.3) it follows that

$$
u(x, \tau)=\frac{N}{\sqrt{4 \pi \tau}}\left(\exp \left(-\frac{\left(x-x_{0}\right)^{2}}{4 \tau}\right)-\exp \left(-\frac{\left(x+x_{0}\right)^{2}}{4 \tau}\right)\right),
$$

with total flux

$$
J(\tau)=\left.\int_{0}^{\tau} \frac{\partial u\left(x, \tau^{\prime}\right)}{\partial x}\right|_{x=0} d \tau^{\prime}=\frac{N x_{0}}{2 \sqrt{\pi}} \int_{0}^{\tau}{\frac{1}{\tau^{\prime}}}^{\frac{3}{2}} \exp \left(-\frac{x_{0}^{2}}{4 \tau^{\prime}}\right) d \tau^{\prime} .
$$

By substituting $z=\frac{x_{0}}{\sqrt{4 \tau}}$ the integral reduces to

$$
\begin{gathered}
J(\tau)=\frac{2 N}{\sqrt{\pi}} \int_{\frac{x_{0}}{\sqrt{4 \tau}}}^{\infty} \exp \left(-z^{2}\right) d z=N\left(1-\operatorname{erf}\left(\frac{x_{0}}{\sqrt{4 \tau}}\right)\right) \\
\Rightarrow J(\tau)=N \operatorname{erfc}\left(\frac{x_{0}}{\sqrt{4 \tau}}\right) \quad \text { with } \quad \tau=\int_{0}^{t} D\left(t^{\prime}\right) d t^{\prime} .
\end{gathered}
$$

Here, $\operatorname{erfc}(z)$ is the complimentary error function with relation $\operatorname{erfc}(z)=1-\operatorname{erf}(z)$. The analytic expressions (A.6) and (A.8) represent the total flux for initial uniform distribution and PSR, respectively.

(III) More generally, for an arbitrary initial distribution $\psi(x)$,

$$
\begin{aligned}
J(\tau)= & \left.\int_{0}^{\tau} \frac{1}{\sqrt{4 \pi \tau^{\prime}}} \int_{0}^{\infty} \psi(\hat{x}) \frac{\partial G\left(x, \hat{x}, \tau^{\prime}\right)}{\partial x}\right|_{x=0} d \hat{x} d \tau^{\prime} \\
& \Rightarrow J(\tau)=\int_{0}^{\infty} \psi(\hat{x}) \operatorname{erfc}\left(\frac{\hat{x}}{\sqrt{4 \tau}}\right) d \hat{x} .
\end{aligned}
$$




\section{B. Simulated trap counts at discrete times $t_{i}=\frac{i T}{p}$}

TABLE 1. Simulated trap counts $J_{C}^{r}\left(t_{i}\right)$ for Cauchy type random walkers for $\mathbf{k}=\mathbf{5} \times \mathbf{1 0}^{-\mathbf{5}}$ over $r=3$ realizations, at discrete times $t_{i}=\frac{i T}{p}, i=1,2, \ldots, p=20$. Other typical parameters include $U_{0}=N=10^{4}, \eta=1$. Here, $T=1.7653$ at which $m=0.7$ i.e $70 \%$ of total trap counts are registered after the first simulation run.

\begin{tabular}{cccccc}
\hline \hline$i$ & $t_{i}$ & $J_{C}^{1}$ & $J_{C}^{2}$ & $J_{C}^{3}$ & $\left\langle J_{C}^{r}\right\rangle$ \\
\hline 1 & 0.0882 & 1018 & 1087 & 1043 & 1049.33 \\
2 & 0.1765 & 1741 & 1775 & 1770 & 1762 \\
3 & 0.2647 & 2359 & 2378 & 2420 & 2385.67 \\
4 & 0.3530 & 2826 & 2916 & 2931 & 2891 \\
5 & 0.4413 & 3261 & 3394 & 3374 & 3343 \\
6 & 0.5295 & 3667 & 3808 & 3790 & 3755 \\
7 & 0.6178 & 4046 & 4157 & 4140 & 4114.33 \\
8 & 0.7061 & 4405 & 4484 & 4465 & 4451.33 \\
9 & 0.7943 & 4662 & 4782 & 4778 & 4740.67 \\
10 & 0.8826 & 4951 & 5088 & 5042 & 5027 \\
11 & 0.9709 & 5224 & 5374 & 5296 & 5298 \\
12 & 1.0591 & 5489 & 5604 & 5519 & 5537.33 \\
13 & 1.1474 & 5716 & 5839 & 5741 & 5765.33 \\
14 & 1.2357 & 5953 & 6042 & 5965 & 5986.67 \\
15 & 1.3239 & 6150 & 6236 & 6197 & 6194.33 \\
16 & 1.4122 & 6334 & 6429 & 6394 & 6385.67 \\
17 & 1.5005 & 6512 & 6597 & 6589 & 6566 \\
18 & 1.5887 & 6693 & 6761 & 6763 & 6739 \\
19 & 1.6770 & 6840 & 6910 & 6902 & 6884 \\
$p=20$ & 1.7653 & 7000 & 7062 & 7045 & 7035.67
\end{tabular}

TABLE 2. Simulated trap counts $J_{C}^{r}\left(t_{i}\right)$ for Cauchy type random walkers for $\mathbf{k}=\mathbf{8} \times \mathbf{1 0}^{-\mathbf{5}}$ over $r=3$ realizations, at discrete times $t_{i}=\frac{i T}{p}, i=1,2, \ldots, p=20$. Other typical parameters include $U_{0}=N=10^{4}, \eta=1$. Here, $T=1.0941$ at which $m=0.7$ i.e $70 \%$ of total trap counts are registered after the first simulation run.

\begin{tabular}{cccccc}
\hline \hline$i$ & $t_{i}$ & $J_{C}^{1}$ & $J_{C}^{2}$ & $J_{C}^{3}$ & $\left\langle J_{C}^{r}\right\rangle$ \\
\hline 1 & 0.0547 & 1045 & 1083 & 1071 & 1066.33 \\
2 & 0.1094 & 1765 & 1804 & 1809 & 1792.67 \\
3 & 0.1641 & 2332 & 2399 & 2440 & 2390.33 \\
4 & 0.2188 & 2864 & 2922 & 2936 & 2907.33 \\
5 & 0.2735 & 3314 & 3371 & 3415 & 3366.67 \\
6 & 0.3283 & 3712 & 3770 & 3838 & 3773.33 \\
7 & 0.3829 & 4062 & 4091 & 4199 & 4117.33 \\
8 & 0.4376 & 4421 & 4426 & 4529 & 4458.67 \\
9 & 0.4924 & 4737 & 4743 & 4829 & 4769.67 \\
10 & 0.5471 & 5037 & 5031 & 5108 & 5058.67 \\
11 & 0.6017 & 5276 & 5304 & 5376 & 5318.67 \\
12 & 0.6565 & 5512 & 5542 & 5626 & 5560.00 \\
13 & 0.7112 & 5739 & 5772 & 5865 & 5792.00 \\
14 & 0.7659 & 5929 & 5971 & 6086 & 5995.33 \\
15 & 0.8206 & 6131 & 6190 & 6291 & 6204.00 \\
16 & 0.8753 & 6314 & 6386 & 6464 & 6388.00 \\
17 & 0.9300 & 6500 & 6574 & 6663 & 6579.00 \\
18 & 0.9847 & 6658 & 6750 & 6834 & 6747.33 \\
19 & 1.0394 & 6839 & 6898 & 7000 & 6912.33 \\
$p=20$ & 1.0941 & 7000 & 7053 & 7141 & 7064.67
\end{tabular}




\section{References}

[1] D. Ahmed. Stochastic and Mean field approaches for trap count modelling and interpretation. PhD thesis, Leicester University, (2014).

[2] R. Balescu. Equilibrium and Non-equilibrium Statistical Mechanics. New York: John Wiley, 1975.

[3] F. Bartumeus, J. Catalan, G. Viswanathan, E. Raposo, M. da Luz. The influence of turning angles on the success of non-oriented animal searches. J. Theor. Biol., 252 (2008) $43-55$.

[4] B. Berkowitz, H. Scher. Theory of anomalous chemical transport in random fracture networks. Phys. Rev. E, 57 (1998) $5858-5869$.

[5] I. Blumen, J. Klafter. Drude approach to anomalous diffusion: Application to richardson dispersion in turbulent flows. Europhys. Lett., 47 (1999) $152-157$.

[6] J. Bouchaud, A. Georges. Anomalous diffusion in disordered media: Statistical mechanics, models and physical applications. Phys. Rep., 195 (1990) $127-293$.

[7] P. Bovet, S. Benhamou. Spatial analysis of animals movements using a correlated random walk model. J. Theor. Biol., 131 (1988) $419-433$.

[8] K. Burnham, D. Anderson. Model selection and multimodel inference: a practical information theoretical approach. Springer, 2002.

[9] S. Cantrell, C. Cosner. Spatial ecology via reaction and diffusion equations. Wiley John and Sons, 2003.

[10] J. Q. Chambers, N. Higuchi, J. P. Schimel. Ancient trees in amazonia. Nature, 391 (1998) $135-136$.

[11] E. Charnov. Life History Invariants: Some Explorations of Symmetry in Evolutionary Ecology. Oxford Univ. Press, 1993.

[12] E. Codling, M. Plank, S. Benhamou. Random walk models in biology. J. R. Soc. Interface, 5 (2008) 813-834.

[13] S. Coscoy, E. Huguet, F. Amblard. Statistical analysis of sets of random walks: how to resolve their generating mechanism. Bull. Math. Biol., 69 (2007) 2467 - 2492.

[14] J. Crank. The mathematics of diffusion. Oxford Univ. Press, 2nd edn., 1975.

[15] P. Davis, P. Rabinowitz. Methods of numerical integration. New York: Academic Press, 1975.

[16] M. De Jager, F. Weissing, P. Herman, B. Nolet, J. vande Koppel. Response to comment on Lévy walks evolve through interaction between movement and environmental complexity. Science, 335 (2012) 918.

[17] J. Dennis, D. Gay, R. Walsh. An adaptive non-linear least-squares algorithm. ACM Trans. Math. Soft., 7 (1981) 348 $-368$.

[18] J. Durbin. Testing for serial correlation in least squares regression. Biometrika, 37 (1950) $409-428$.

[19] N. Embleton, N. Petrovskaya. A novel approach to evaluation of pest insect abundance in the presence of noise. Bull. Math. Biol., 76 (2014) $718-743$.

[20] A. Fick. Ueber diffusion. Annalen der Physik, 170 (1855) 59 - 86.

[21] R. Fisher. The wave of advance of advantageous genes. Annals of Eugenics, 7 (1937) $355-369$.

[22] L. Giuggioli, F. Sevilla, V. Kenkre. A generalized master equation approach to modelling anomalous transport in animal movement. J. Phys., 42 (2009) $434-444$.

[23] N. Gotelli, A. Ellison. A primer of ecological statistics. Sunderland: Sinauer Associates, 2004.

[24] J. P. Grime, R. Hunt. Relative growth-rate: its range and adaptive significance in a local flora. Ecology, 63 (1975) 393 -422 .

[25] R. S. Grimm, V. Individual based modelling and Ecology. Princeton Univ. Press, 2005.

[26] R. Grimmet, D. Stirzaker. Probability and random processes. Oxford Univ. Press, 2001.

[27] E. Holmes, M. Lewis, J. Banks, R. Veit. Partial differential equations in ecology: spatial interactions and population dynamics. Ecology, 75 (1994) $17-29$.

[28] V. Jansen, A. Mashanova, S. Petrovskii. Model selection and animal movement: comment on Lévy walks evolve through interaction between movement and environmental complexity. Science, 335 (2012) 918.

[29] F. Jopp, H. Reuter. Dispersal of carabid beetles: emergence of distribution patterns. Ecol. Model., 186 (2005) 389 405.

[30] P. Kareiva. Local movement in herbivorous insects: applying a passive diffusion model to mark-recapture field experiments. Oecologia, 57 (1983) $322-327$.

[31] P. Kareiva, N. Shigesada. Analyzing insect movement as a correlated random walk. Oecologia, 56 (1983) $234-238$.

[32] R. Kawai. Continuous time modeling of random searches: Statistical properties and inference. J. Phys. A: Math. Theor., 45 (2012) 235004.

[33] M. Kot, M. Lewis, P. Van der Driessche. Dispersal data and the spread of invading organisms. Ecology, 77 (1996) 2027 -2042 .

[34] H. Malchow, S. Petrovskii, E. Venturino. Spatiotemporal patterns in ecology and epidemiology: theory, models, and simulations. Chapman Hall/CRC, 2008.

[35] R. Metzler, J. Klafter. The random walks guide to anomalous diffusion: a fractional dynamics approach. Phys. Rep., 339 (2000) $1-77$.

[36] R. Metzler, J. Klafter. The restaurant at the end of the random walk: Recent developments in the description of anomalous transport by fractional dynamics. J. Phys. A: Math. Gen., 37 (2004) $161-208$.

[37] E. Montroll, G. Weiss. Random walks on lattices. J. Math. Phys., 6 (1965) $167-181$. 
[38] J. Morales, D. Haydon, J. Frair, K. Holsinger, J. Fryxell. Extracting more out of relocation data: building movement models as mixtures of random walks. Ecology, 85 (2004) $2436-45$.

[39] N. Newlands, M. Lutcavage, T. Pitcher. Analysis of foraging movements of atlantic blue fin tuna (thunnus thynnus): individuals move with two modes of searching. Popul. Ecol., 46 (2004) 39 - 53.

[40] A. Okubo. Diffusion and ecological problems. Springer, New York, 1980.

[41] A. Okubo, H. Chiang. An analysis of the kinematics of swarming of anarete pritchardi kim (diptera: Cecidomyiidae). Res. Popul. Ecol., 16 (1974) $1-42$.

[42] A. Okubo, S. Levin. Diffusion and ecological problems: modern perspectives. Springer, New York, 2001.

[43] A. Okubo, S. Levin. Some examples of animal diffusion. Springer-Verlag, 2001.

[44] J. Patrick. Computational fluid dynamics. Hermosa Publishers, 1976.

[45] N. Petrovskaya, S. Petrovskii, A. Murchie. Challenges of ecological monitoring: estimating population abundance from sparse trap counts. J. R. Soc. Interface, 9 (2011) 420-435.

[46] S. Petrovskii, D. Ahmed, R. Blackshaw. Estimating insect population density. Ecol. Complexity, 10 (2012) 69-82.

[47] S. Petrovskii, L. Brian. Exactly solvable models of biological invasion. Chapman and Hall/CRC, 2006.

[48] S. Petrovskii, A. Mashanova, V. Jansen. Variation in individual walking behavior creates the impression of a Lévy flight. Proc. Natl. Acad. Sci. USA., 108 (2011) $8704-8707$.

[49] S. Petrovskii, A. Morozov. Dispersal in a statistically structured population. Am. Nat., 173 (2008) $278-289$.

[50] S. Petrovskii, N. Petrovskaya, D. Bearup. Multiscale approach to pest insect monitoring: Random walks, pattern formation, synchronization and networks. Phys. Rev., 11 (2014) $467-525$.

[51] M. Plank, E. Codling. Sampling rate and misidentification of Lévy and non-Lévy movement paths. Ecology, 90 (2009) $3546-3553$.

[52] G. Radons, R. Klages, I. Sokolov. Anomalous transport. Berlin: Wiley-VCH, 2008.

[53] A. Reynolds. Scale-free animal movement patterns: Lévy walks outperform fractional Brownian motions and fractional Lévy motions in random search scenarios. J. Phys. A: Math. Theor., 42 (2009) 434006.

[54] A. Reynolds. Bridging the gulf between correlated random walks and Lévy walks: autocorrelation as a source of Lévy walk movement patterns. J. R. Soc. Interface, 7 (2010) 1753 - 1758.

[55] P. Richards. The tropical rainforest. Cambridge Univ. Press, 2nd edn, 1996.

[56] L. Richardson. Atmospheric diffusion shown on a distance-neighbour graph. Proc. R. Soc. London: Series A, 110 (1926) $709-737$.

[57] H. Scher, M. Lax. Stochastic transport in a disordered solid. Phys. Rev. B, (1973) $4491-4502$.

[58] H. Scher, E. Montroll. Anomalous transit-time dispersion in amorphous solids. Phys. Rev. B, 12 (1975) 2455 - 2477.

[59] H. Scher, M. Shlesinger, J. Bendler. Time-scale invariance in transport and relaxation. Phys. Today, 44 (1991) $26-$ 34.

[60] N. Shigesada, K. Kawasaki. Biological invasions: theory and practice. Oxford Univ. Press, 1997.

[61] N. Shigesada, K. Kawasaki. Invasion and the range expansion of species: effects of long distance dispersal. Dispersal Ecology, (2002) 350 - 373.

[62] M. Shlesinger. Asymptotic solutions of continuous-time random walks. J. Stat. Phys., 10 (1974) $421-434$.

[63] M. Shlesinger. Random searching. J. Phys. A: Math. Theor., 42 (2009) 434001.

[64] J. Skellem. Random dispersal in theoretical populations. Biometrika, 38 (1951) $196-218$.

[65] G. Smith. Numerical Solution of Partial Differential Equations: Finite Difference Methods (Oxford Applied Mathematics Computing Science Series). Oxford Univ. Press, 1986.

[66] S. Stearns. The Evolution of Life Histories. Oxford Univ. Press, 1992.

[67] V. Stern. Economic thresholds. Entomol., 18 (1973) 259-280.

[68] W. Strauss. Partial differential equations: An introduction. John Wiley and Sons, 2008.

[69] P. Tilles, S. Petrovskii. Statistical mechanics of animal movement: Animal's decision making can result in superdiffusive spread. Ecol. Complexity, (2015). In Press, http://dx.doi.org/10.1016/j.ecocom.2015.02.006.

[70] D. Tilman. Plant Strategies and the Dynamics and Structure of Plant Communities. Princeton Univ. Press, 1988.

[71] P. Turchin. Quantitative analysis of movement: measuring and modelling population redistribution in animals and plants. Sinauer Associates, 1998.

[72] G. Viswanathan, V. Afanasyev, S. Buldryrev, S. Havlin, M. da Luz, E. Raposo, H. Stanley. Lévy flights in random searches. Phys. A, 282 (2000) 1-12.

[73] G. Viswanathan, V. Afanasyev, S. Buldryrev, S. Havlin, R. da Luz, M., H. Stanley. The Physics of Foraging. Cambridge Univ. Press, 2011.

[74] E. Weeks, J. Urbach, H. Swinney. Anomalous diffusion in asymmetric random walks with a quasi-geostrophic flow example. Phys. D: Nonlinear Phen., 97 (1996) $291-310$.

[75] G. Weiss. Aspects and applications of the random walk. North Holland Press, 1994.

[76] G. Weiss, R. Rubin. Random walks: theory and selected applications. Adv. Chem. Phys., 1983.

[77] G. Zaslavsky, S. Benkadda. Chaos, Kinetics and Non-linear Dynamics in Fluids and Plasmas. Springer, Berlin, 1998. 